\title{
La(s) protección(es) social(es) en Argentina 2020: Lo que la pandemia alumbró
}

Social protection(s) in Argentina 2020: what the pandemic illuminated

\section{Evangelina Benassi y Roberto Zampani}

Evangelina Benassi es docente e investigadora en la Escuela de Trabajo Social, Facultad de Ciencia Política y Relaciones Internacionales, Universidad Nacional de Rosario, Argentina.

E-mail: evangelinabenassi@gmail.com
Roberto Zampani es docente e investigador en la Escuela de Trabajo Social, Facultad de Ciencia Política y Relaciones Internacionales, Universidad Nacional de Rosario, Argentina.

E-mail: rzampani@gmail.com

\section{resumen}

El escenario de pandemia actual reformula la pregunta sobre las "protecciones sociales" para las personas en este contexto. Proponemos recorrer históricamente desde el siglo XX hasta la actualidad las distintas formas de protección social en Argentina, analizando el papel del mundo del "trabajo" en dicho proceso. Asimismo, identificamos las políticas de transferencia de ingresos como la "Asignación Universal por Hijo" (AUH) y el "Ingreso Familiar de Emergencia" (IFE), las cuales se presentan como novedosas formas de "protección social". Por último, recuperamos ciertos interrogantes que el contexto actual presenta y consideramos sus posibles articulaciones en el futuro inmediato.

\section{palabras clave}

pandemia / protecciones sociales / mundo del trabajo / políticas de transferencias de ingresos

\section{keywords}

pandemic / social protections / world of work / income transfer policies 


\section{Introducción}

En este artículo nos proponemos poner en discusión algunos de los debates respecto de las protecciones sociales que cobraron visibilidad en el marco de la pandemia. Como equipo docente de la asignatura "Política social I" de la Licenciatura en Trabajo Social desde hace años nos planteamos la problemática respecto de la crisis del mercado de trabajo y las connotaciones que generan las transformaciones en un sistema de protección que sostiene el empleo como vector central de la integración social (Andrenacci y Soldano, 2006). En ese sentido, partimos de la premisa de que la pandemia no generó un escenario demasiado novedoso en materia de protecciones, sino que, por el contrario, visibilizó lo que se sostiene desde hace más de 30 años en la literatura específica de este campo (Grassi, 2003; Danani, 2009 Hintze y Costa, 2011; Arcidiácomo et al., 2011): que la protección ligada al empleo formal genera y profundiza las inequidades y desigualdades entre los diferentes tipos de trabajo -tanto formal como informal- que la población realiza para poder vivir. Por este motivo, en este caso analizaremos tres cuestiones que reponen ese debate y que nos permiten, luego, pensar e imaginar algunos escenarios futuros.

En un primer punto, repasaremos a grandes rasgos la discusión respecto de las protecciones sociales en el siglo XX, las cuales, centradas en el mercado formal de trabajo, tuvieron que resignificarse con su crisis a mediados de la década de 1970. Si bien estamos hablando de un período de más de 30 años de revisión de las políticas sociales, sus transformaciones institucionales fueron mucho menos profundas que los cambios societales y económicos que se produjeron, lo cual generó cada vez más dificultades en términos de garantizar cobertura y eficacia de las protecciones.

En un segundo punto, retomamos sintéticamente la propuesta del ingreso ciudadano que surge en los albores del siglo XXI como alternativa a la salida de la mencionada crisis de las protecciones. En este sentido, veremos de qué modo su viabilidad política en Latinoamérica derivó en políticas de transferencias monetarias condicionadas. Dichas transferencias se expresaron, en el caso argentino, mediante la Asignación Universal por Hijo (AUH), mojón que, consideramos, deja una huella ineludible para pensar en las reformas actuales del sistema de políticas sociales.

Por último, pensaremos el escenario actual y la centralidad que el Ingreso Familiar de Emergencia (IFE) cobra en este contexto. Para ello, realizaremos un punteo de cuáles serían algunas posibilidades de articulación de políticas que generen una mayor integralidad en la protección social, de cara a un escenario caracterizado por la incertidumbre.

\section{Intentar, lo intentamos...}

Pueden venir cuantos quieran, que serán tratados bien los que estén en el camino, bienvenidos al tren Sui Generis, "Bienvenidos al tren" 
Analizar el sistema de protecciones sociales en un país requiere repasar tanto el desarrollo histórico, sus avatares económicos, sociales, políticos y culturales, como las distintas decisiones que se tomaron desde el Estado en función de atender las diferentes problemáticas que la cuestión social adoptó en cada momento y en cada región. Así, durante gran parte del siglo XX, podemos identificar la construcción de un sistema de protecciones sociales que, en el caso argentino, pueden ser resumidas en tres grandes campos de políticas, en términos de Andrenacci y Soldano (2006): políticas del trabajo, políticas de provisión de servicios básicos universales y políticas asistenciales.

Podemos entonces identificar que, en el tránsito del siglo XX, desde las primeras luchas obreras hasta el reconocimiento de derechos sociales laborales -sumado a una estructura y desarrollo económico que favoreció el pleno empleo-, las protecciones al trabajo se configuraron como el canal fundamental por el cual el grueso de la población accedió a bienes y servicios que les servían como reaseguro frente a los avatares y riesgos de la vida. El listado de protecciones incluyó sistemas de jubilaciones y pensiones, seguros de desempleo, resguardos frente a despidos, seguros al trabajo, leyes laborales de descanso y protección del salario, así como prestaciones y protecciones a familiares directos del sujeto trabajador. Todas esas políticas fueron configurando en Argentina una estructura social "protegida".

Por otro lado, mediante la combinación de diversas motivaciones políticas, sociales y sanitarias se produjo en nuestro país el desarrollo y la profundización de una serie de servicios básicos universales que aseguraban a la población acceso a bienes y servicios que impactaban de forma directa en la calidad de vida, tanto en el plano individual como en el social. Estas políticas permitieron constituir lo que Andrenacci y Soldano (2006) denominan como "umbral mínimo de ciudadanía". De ese entramado de servicios podemos balizar los dos grandes sistemas públicos por excelencia: el sistema de salud pública y el sistema de educación pública. Asimismo, otros servicios públicos que hacen a la vida urbana -aunque no exclusivamente-, como los servicios de agua potable y cloacas, energías eléctricas, gas, transporte público, entre otros, permitieron mostrar otras formas del sistema de protección y bienestar social en términos generales.

Por último, el tercer campo de políticas previamente mencionado surge a partir de reconocer la existencia de sectores y grupos de la sociedad que requieren una atención especial, ya sea por su dificultad para ser incluidos en los mercados laborales -por edad o condición física-, o bien que requieren una mirada específica por su particularidad. Así, las políticas asistenciales son las que dieron respuesta a las necesidades de estos segmentos, "completando" un sistema de políticas sociales cuya cobertura logró proteger gran parte de la población

Este recorrido, que recién en la década de 1980 empieza a resquebrajarse, nos muestra los diferentes "sujetos de la protección". Podemos identificar, así, políticas que anclan su accionar sobre el "trabajo", en cuyo marco, en específico, las protecciones se obtienen por la condición de trabajador o trabajadora de las personas. Por otro lado, tenemos un sujeto "ciudadano", esto es, políticas de servicios básicos universales que son desarrolladas en torno a la idea de la población como 
ciudadanía y que buscan llegar a los máximos niveles de universalización. Por último, observamos un sujeto que no puede por sí mismo acceder a la categoría trabajador y, a su vez, requiere protecciones sociales específicas que no alcanzan con las meras prácticas universales. El sujeto de las políticas de asistencia transita entre los márgenes de las políticas del trabajo y las políticas de servicios básicos universales. Según el período histórico, este sujeto fue caracterizado de diferentes formas: pobres, menesterosos, vagabundos, planeros.

Si bien el despliegue de estas diferentes políticas sociales significa -en términos analíticos- el reconocimiento de diversos sujetos de las protecciones, el imaginario colectivo se sostiene sobre la certeza de que la protección social debe ser adquirida -cuando no "ganada"- a través del trabajo, y específicamente del empleo. Así, durante todo el siglo XX se fue consolidando la asociación trabajo/ protección social, pareja que deslegitima y quita entidad a las otras dos categorías previamente mencionadas. Quien merece ser protegido, bajo esta perspectiva, es el trabajador, por el esfuerzo que hace para "ganarse la vida".

A su vez, en términos institucionales, cada vez más se acompañó y reforzó la legitimidad de esa dupla y las protecciones fueron incrementando su potencial protector y su cobertura para aquellos que son parte del mercado formal de trabajo, precarizando y/o minimizando las propuestas universalistas y asistenciales.

\section{Mojones que dejaron huellas}

Quedabas esperando ecos que no volverán, flotando entre rechazos

del mismo dolor, vendrá un nuevo amanecer Gustavo Cerati, "Adiós"

Sobre el fin del siglo XX, con el avance de las políticas de ajuste estructural (Gigli, 2006) en el territorio latinoamericano, la denominada crisis del mundo del trabajo y del Estado social en términos de Robert Castel (1997; 2004), sumado al triunfo y diseminación de las políticas de corte neoliberal en los diversos países de Nuestramérica, el edificio de protecciones sociales construido en torno a los tres tipos de políticas que mencionamos presentó grandes debilidades en su institucionalidad y en sus prácticas para atender esas transformaciones. Asimismo, los cambios sociales evidenciaban que los sujetos que se encontraban bajo el resguardo de ese sistema de protecciones también habían mutado, lo que puso de manifiesto, fundamentalmente, un cambio drástico en torno a quién debía constituirse en "sujeto de la asistencia estatal". Si antes se trataba de quien quedaba por fuera del mercado de trabajo, luego la cantidad de población que se encontraba en esa situación era demasiado cuantiosa como para responsabilizarles de "no querer o no poder trabajar". Si bien la lógica de la activación y responsabilización individual (Merklen, 2012) fue la que hegemonizó la lectura de las transformaciones sociales en términos de protecciones sociales, era evidente que esos sujetos "querían trabajar" pero no encontraban soportes institucionales que les permitieran hacerlo.

Además, la consolidación de economías que soportan y mejoran su nivel de desarrollo con altos niveles de desocupación, el cambio y desregulación del mercado 
de trabajo, la diseminación de formas precarias de empleo, así como la privatización de los fondos de jubilación y pensión, generan un escenario que modifica drásticamente tanto la sociedad como el perfil de la población a proteger. Tal como decíamos previamente, si durante gran parte del siglo XX la sociedad estaba organizada alrededor de la figura del "trabajador", a partir de los cambios antes mencionados las políticas e intervenciones estatales comienzan a concentrarse en otras figuras. Esta novedad no fue ingenua: "precarizar" y empobrecer a los trabajadores fue una estrategia que permitía sostener la ideología individualizante previamente mencionada. Así, el sujeto de las intervenciones estatales pasa a definirse como "nuevos pobres" o en algunos casos "empobrecientes", negando que ese universo estaba conformado por "trabajadores sin trabajo" (Castel, 1997). En palabras de Merklen (2005), se da un proceso de conversión de "trabajadores a pobres".

Asimismo, las políticas de servicios básicos universales experimentaron un cambio institucional fuerte a partir de la descentralización de sus prestaciones -transferencias de responsabilidades nacionales a los niveles subnacionales provinciales y municipales de los sistemas de educación y salud pública con casi nula transferencia de recursos-y la privatización en las empresas prestadoras de servicios públicos (agua, energía eléctrica, gas, telefonía).

En este contexto de transformaciones de las políticas referidas al trabajo y su protección, y en el marco de un cambio radical en las políticas de servicios básicos universales, las políticas asistenciales adquieren una nueva dimensión las políticas asistenciales y se convierten en uno de los ejes privilegiados de las intervenciones "sociales" del Estado. Esto se debe, además, al hecho de que si antes atendían a una población marginal, terminaron por convertirse en el centro de las protecciones sociales. Es posible identificar aquí dos grandes caminos que, consideramos, dejaron mojones que nos permiten problematizar la contemporaneidad: los planes y políticas de atención a la situación alimentaria y las políticas de transferencia de ingresos.

Desde la recuperación democrática en Argentina, el tema "alimentario" pasa a ocupar un lugar de protagonismo en las áreas de asistencia/promoción/desarrollo social de los diversos niveles del Estado. Podríamos trazar una línea histórica desde el Plan Alimentario Nacional en el año 1984 hasta la Tarjeta Alimentar en 2020, pasando por la distribución masiva de alimentos en niveles provinciales y municipales, como también otras experiencias de asignación masiva de "bonos alimentarios" o "tickets alimentarios". Esta línea nos muestra un problema que, a pesar de que fue periférico en el sistema estatal de protección y bienestar social argentino -casi siempre abordado por iglesias y entes de caridad, beneficencia o filantropía-, pasa a ocupar un lugar importante en la organización pública estatal. Tanto en el nivel presupuestario como en la tecnoburocracia necesaria para su puesta en práctica, el tema "alimentario" ocupa gran parte de la agenda diaria de los diversos niveles del Estado. Esta transformación también impacta y resignifica otras instituciones sobre las cuales se asentaba el sistema de protección social, como lo es la "familia tradicional". En ese sentido, la proliferación de los comedores comunitarios en la década de 1980 generó diversas posiciones que 
auguraban la degradación social que significaría el fin del "almuerzo compartido en familia".

Podemos pensar que los planes alimentarios trazan una línea de continuidad con la vieja lógica asistencial porque recortan como población la denominada "pobreza estructural" o aquellas familias de sectores populares que históricamente oscilaron entre la protección vía el trabajo informal y la asistencia. En ese sentido, no plantea grandes novedades en términos de la definición del sujeto al cual va dirigida, pero sí en relación con la dimensión de su cobertura. De hecho, las políticas alimentarias siempre estuvieron controladas socialmente desde la mirada disciplinadora del sentido común que plantea "mejor que les den [a los pobres] alimentos y no dinero, porque si no es muy probable que lo usen en alcohol y juego".

Sin embargo, por otro lado, en el marco de las transformaciones estructurales mencionadas al comienzo de este apartado, hacen su aparición en escena las denominadas "políticas de transferencias de ingresos". ¿Cuáles son las grandes novedades que estas políticas plantean? Por un lado, la visibilización del lugar que los ingresos tienen en la satisfacción de las necesidades (Bauman, 2005 y, por otro, se entiende que la búsqueda de cierto nivel de bienestar para determinados sectores de la población viene dada por la transferencia periódica, organizada y sistemática de dinero. En ese sentido, el debate que opera como telón de fondo es el que comienza a gestarse en los países escandinavos de la mano de Van Parijs (1995) y que plantea la necesidad de institucionalizar un ingreso universal e incondicional a todos los ciudadanos para la protección social. Esta propuesta reconoce las transformaciones del capitalismo a escala global y la necesidad de contrarrestar-desde la lógica monetaria del propio sistema- su dinámica excluyente y destructora de los mecanismos de integración social.

Así, estas propuestas parten de considerar que las transformaciones del mercado de trabajo son de índole estructural y que incluso es ecológicamente inviable la restauración de esa vieja lógica de protección social, por lo cual el Estado debe garantizar un mínimo de protección a través de la emisión monetaria. A su vez, la propuesta también busca visibilizar que los sujetos que requieren la asistencia monetaria estatal "quieren trabajar", buscan trabajo y no logran conseguirlo. Es por eso que la propuesta del ingreso ciudadano universal se considera como una vía institucionalizada para reconocer las transformaciones que se produjeron en los últimos 30 años del siglo XX.

Sin embargo, la viabilidad política de este tipo de propuestas universalistas e incondicionales siempre fue precaria en Latinoamérica porque, tal como planteamos previamente, la valoración moral del "trabajo" como medio "digno" para ganarse la vida opera como el gran regulador social.

De acuerdo con Midaglia (2012), podemos reconocer que las traducciones de estas propuestas se conocen en Latinoamérica, desde mediados de la década de 1990, como políticas de transferencias de ingresos condicionadas (Transferencias condicionadas de renta, en términos de la autora). Las propuestas se centran en atender grupos poblacionales específicos y focalizados debido a la dificultad de establecer un consenso político e institucional que legitime políticas de trans- 
ferencias universales. Estas propuestas consisten en prestaciones monetarias para personas y/o familias, a las que se les solicita a cambio el compromiso o "condicionalidad" de atender o cumplir con las protecciones sociales básicas del grupo familiar: las dimensiones de salud y educación, especialmente de niños y niñas.

Este fenómeno -que a nivel continental reconoce las experiencias del "Bolsa Familia" en Brasil y "Oportunidades" en México- se institucionaliza en nuestro país, a partir del año 2009, con la implementación de la "Asignación Universal por Hijo para Protección Social" (AUH), complementada desde 2011 con la "Asignación Universal por Embarazo para Protección Social" (AUE). Esta intervención estatal, anclada en el sistema de asignaciones familiares ${ }^{1}$, tiene como sujetos de la protección a niños, niñas y adolescentes cuyos padres o tutores estén desocupados o se desempeñen en la economía informal por un salario menor al mínimo vital y móvil (Arcidiacomo et al., 2011).

La AUH se reconoce como heredera del debate por el ingreso ciudadano y universal, pero, si se considera la viabilidad política, la manera de poder llevarla adelante fue poner el eje en la figura de los niños y niñas y, a través de ellos, generar protecciones familiares, con la consideración de que sus padres trabajan en empleos precarios (Hintze y Costa, 2011). En ese sentido, la AUH logró un alto nivel de consenso social y, si bien al comienzo de su implementación se generaron diversos y acalorados debates que ponían el foco en la debilidad que las condicionalidades generaban, hoy es una política que se encuentra legitimada socialmente.

Tal magnitud se observa en el mapa aproximado de niños y niñas que se encuentran cubiertos por la protección de la AUH: en junio de 2019, casi cuatro millones (3.923.040) de niños y niñas eran titulares de derechos de AUH, a cargo de un total de padres, madres o tutores que asciende a 2.208.323. A estas cifras, hay que añadir casi 78.000 Asignaciones Universales por Embarazo (AUE) para el mismo mes. ${ }^{2}$ Esta dimensión y alcance la convierte en una de las experiencias de transferencia de ingresos o renta de mayor nivel y aspiración universal en nuestro país.

\section{Lo que la pandemia visibilizó}

Un tornado arrasó a mi ciudad y a mi jardín primitivo, un tornado arrasó a tu ciudad y a tu jardín primitivo... Sumo, "Mejor no hablar de ciertas cosas"

Pero un día, ¿todo cambió? La crisis sanitaria, social y económica que se produjo durante 2020, como consecuencia de la expansión de la pandemia por el virus Covid-19 -vulgarmente conocido como "coronavirus"-, afecta de diversas maneras a nuestro continente, a nuestros países, a nuestras ciudades y barrios. Todos los ámbitos de la vida entraron en crisis. La totalidad de la vida en sociedad se encuentra en una situación de "suspensión". La realidad económica muestra caras desconocidas. La forma de relacionarnos es interrogada y cuestionada. Cambia la forma de movernos, de cuidarnos, la diversión, la cultura, el esparcimiento. 
No solamente son interpelados los gobiernos y sus formas de gobernar. También es interpelada la sociedad y sus instituciones; las personas en general estamos en la búsqueda de afrontar y descubrir nuevas herramientas para encarar el mundo que viene. Vivimos en una situación general de incertidumbre: nadie tiene certezas sobre la pandemia, ni sobre su duración en el tiempo, ni su forma de intervenir o enfrentarla. Esta incertidumbre es global, y es una incertidumbre a nivel social.

Ahora bien, en materia de protección social nos preguntamos, ¿qué hay de nuevo en este escenario? El recorrido histórico que trazamos previamente nos permite inferir que algunas de las transformaciones que se visibilizan en el contexto de pandemia dan cuenta de procesos de larga data. La metamorfosis del mundo del trabajo y sus consecuencias en una estructura de protección social que giraba en torno a su formalidad no es un fenómeno nuevo, o que la pandemia haya instalado. A pesar de esto, en un contexto en el cual la precariedad se pone en juego en todos los ámbitos de la vida cotidiana, nos planteamos cuáles son las formas que deben afrontar las protecciones sociales en esta nueva realidad.

Así, la gran novedad la constituye el Ingreso Familiar de Emergencia (IFE), la política social más importante que fue formulada y ejecutada en el contexto de la pandemia. El IFE constituye una apuesta que permite reconocer la diversidad de sujetos que requieren de la intervención del Estado para contar con un mínimo de protección y, a su vez, resignifica cuál es el público legítimo de la asistencia estatal. Si bien el IFE tiene varias debilidades, en tanto se centra en los ingresos ${ }^{3} \mathrm{y}$, además, se plantea como compensatoria o transitoria (oficialmente es descripta como "una prestación monetaria no contributiva de carácter excepcional"4), creemos que su gran aporte es lo que venimos planteando desde el comienzo de este artículo: poner sobre el escenario común, colectivo, la magnitud de la precariedad en la que viven aquellos ciudadanos y ciudadanas que realizan trabajos con escaso reconocimiento y legitimidad tanto social como institucional en las sociedades contemporáneas. ${ }^{5}$ En ese sentido, la cobertura potencial del IFE constituye un dato contundente: 14,2 millones de ciudadanos se encuentran agrupados en esa condición.

Si bien el IFE es un programa anclado en la noción de trabajo - y en la imposibilidad de desarrollarlo-, creemos que su implementación reposicionó un debate que se vuelve central en las sociedades contemporáneas: quién merece ser protegido y de qué manera esto puede viabilizarse. En ese sentido, el ingreso ciudadano vuelve a aparecer en la escena académica y política, pero con la novedad de que esta vez cuenta con un antecedente que ejemplifica la magnitud de la población que queda a "merced de su propio esfuerzo" para poder sobrevivir cotidianamente.

Creemos, entonces, que la pandemia logró reponer un debate que es central en materia de protección, y que habíamos postergado por sucesivas -e inestablesrecuperaciones económicas que volvían a agitar la ilusión de sociedades de pleno empleo y protegidas. Quizás lo que la pandemia nos permita sea repensarnos como sociedad, ampliando las dimensiones materiales y morales respecto de la protección social.

Podríamos arriesgar, como horizonte inmediato, que las protecciones sociales en nuestro país deberían contemplar un sistema que abarque: a) políticas e inter- 
venciones sociales de corte universal fuertes, robustas y masivas -véase la importancia de sistemas de salud pública con recursos materiales y humanos preparados, sistemas educativos actualizados para un mundo híbrido entre lo virtual y lo presencial, así como intervenciones en infraestructura de hábitat y servicios-; b) políticas de transferencias de ingresos con aspiración a cobertura universal frente al contexto desarrollado anteriormente; c) políticas de trabajo que busquen regular e incluir mejoras en las formas de protección al conjunto del trabajo precarizado; y d) desarrollo de un sistema de cuidados que aborde las desigualdades tanto en términos económicos como de género. ${ }^{6}$

Si parafraseamos la serie Dark (Alemania, 2017), podríamos sostener que la pregunta no es inexorablemente dónde, cómo o a quiénes proteger, sino que la pregunta es cuándo. La respuesta está a la vista, esperemos contribuir con esa respuesta.

\section{Referencias}

1. Es para destacar que uno de los factores de "posibilidad" de la AUH, en el marco institucional de las asignaciones familiares bajo responsabilidad de la Administración Nacional de la Seguridad Social, fue la reestatizacion de los fondos de jubilación, previamente privatizadas en 1993 bajo la figura de las Administradoras de Fondos de Jubilación y Pensiones (AFJP), que volvieron al Estado en el año 2008 bajo un nuevo sistema de reparto estatal de jubilaciones y pensiones.

2. Datos de junio de 2019, disponibles en: https://www.anses.gob.ar/informacion/datos-abiertos-asignaciones-universales

3. Un debate que excede la posibilidad de este escrito, pero que es necesario mencionar, tiene que ver con la necesidad de que las políticas sociales combinen propuestas ligadas al incremento de los ingresos con la institucionalización de otro tipo de protecciones, de tipo estructural, tales como salud, educación, vivienda, transporte, alimentación. La medición de la pobreza teniendo como eje la línea de pobreza -canasta básica- ha generado en los últimos años que las estrategias de mejora de esa situación se focalicen más en los ingresos que en otro tipo de bienes y servicios que también son centrales para garantizar una "vida digna".

4.Véase http://observatorio.anses.gob.ar/archivos/documentos/Boletin\%20IFE\%20I-2020.pdf

5. En el documento de ANSES, la potencial población beneficiaria del IFE se agrupa en las siguientes categorías: inactivas (5,8 millones) los/las desempleados/as que no cobran Seguro de Desempleo (1,7 millones), los/as asalariados/as informales (4,5 millones), los/as cuentapropistas informales (alrededor de 2,1 millones, representando una gran proporción de aquellos/as que tienen bajos ingresos), y los/ las trabajadores/as sin remuneración familiar ( 0,1 millones). Fuente: http://observatorio.anses.gob.ar/ archivos/documentos/Boletin\%20IFE\%20I-2020.pdf

6.Si bien excede las posibilidades de este artículo y sería materia de otro ensayo, es necesario y pertinente destacar que las alusiones a "el trabajador" o "el ciudadano" en masculino no son casuales, sino que más bien buscan evidenciar que el sistema de protección social se estructuró (y reforzó) sobre la división sexual del trabajo, poniendo el acento en el "trabajo asalariado realizado por varones como jefes de hogar", desconociendo y desprotegiendo el trabajo femenino no remunerado y la diversidad de trabajos que realizan las mujeres para sostener sus hogares.

\section{Bibliografía}

Andrenacci, L. y Soldano, D. (2006). Aproximación a las teorías de la política social a partir del caso argentino. En L. Andrenacci (Comp.), Problemas de la política social en la Argentina contemporánea . Buenos Aires, Argentina: Prometeo.

Arcidiacomo, P. et al. (2011). La asignación universal por hijo para protección social: ¿rupturas y 
continuidades, hacia un esquema universal? Revista Margen, $\mathrm{N}^{\circ} 61$.

Bauman, Z. (2005). Trabajo, consumismo y nuevos pobres. Barcelona, España: Gedisa.

Castel, R. (1997). Las metamorfosis de la cuestión social. Una crónica del asalariado. Buenos Aires, Argentina: Paidós.

Castel, R. (2004). La inseguridad social. ¿Qué es estar protegido? Buenos Aires, Argentina: Manantial.

Danani, C. (2009). La gestión de la política social: un intento de aportar a su problematización. En M. Chiara y M. M. Di Virgilio (Orgs.), Gestión de la política social. Buenos Aires, Argentina: Prometeo. Grassi, E. (2003). Políticas y problemas sociales en la sociedad neoliberal. Buenos Aires, Argentina: Espacio Editorial.

Hintze, S. y Costa, M. (2011). La reforma de las asignaciones familiares 2009: aproximación al proceso político de la transformación de la protección. En C. Danani y S. Hintze (Coords.), Protecciones y desprotecciones: la seguridad social en la Argentina 1990-2010. Los Polvorines, Argentina: Universidad Nacional de General Sarmiento.

Merklen, D. (2005). Pobres ciudadanos: las clases populares en la era democrática 1983 - 2003. Buenos Aires, Argentina: Editorial Gorla.

Midaglia, C. (2012). Un balance crítico de los programas sociales en América Latina: Entre el liberalismo y el retorno del Estado. Nueva Sociedad, 239, mayo-junio.

Van Parijs, P. (1995). Más allá de la solidaridad. Los fundamentos éticos del Estado de Bienestar y de su superación. En R. Lo Vuolo (Comp.), Contra la exclusión. La propuesta del ingreso ciudadano. Buenos Aires, Argentina: CIEPP - Miño y Dávila.

\section{Fuentes consultadas}

https://www.anses.gob.ar/informacion/datos-abiertos-asignaciones-universales

http://observatorio.anses.gob.ar/archivos/documentos/Boletin\%20IFE\%20I-2020.pdf

Evangelina Benassi y Roberto Zampani, “La(s) protección(es) social(es) en Argentina 2020: Lo que la pandemia alumbró". Revista Temas y Debates. ISSN 1666-0714, año 24, número especial, juliodiciembre 2020, pp. 203-212. 